\title{
Effect of an oscillating time-dependent pressure gradient on Dean flow: transient solution
}

\author{
Basant K. Jha and Dauda Gambo* (1)
}

\begin{abstract}
Background: Navier-Stokes and continuity equations are utilized to simulate fully developed laminar Dean flow with an oscillating time-dependent pressure gradient. These equations are solved analytically with the appropriate boundary and initial conditions in terms of Laplace domain and inverted to time domain using a numerical inversion technique known as Riemann-Sum Approximation (RSA). The flow is assumed to be triggered by the applied circumferential pressure gradient (azimuthal pressure gradient) and the oscillating time-dependent pressure gradient. The influence of the various flow parameters on the flow formation are depicted graphically. Comparisons with previously established result has been made as a limit case when the frequency of the oscillation is taken as $0(\omega=0)$.

Results: It was revealed that maintaining the frequency of oscillation, the velocity and skin frictions can be made increasing functions of time. An increasing frequency of the oscillating time-dependent pressure gradient and relatively a small amount of time is desirable for a decreasing velocity and skin frictions. The fluid vorticity decreases with further distance towards the outer cylinder as time passes.

Conclusion: Findings confirm that increasing the frequency of oscillation weakens the fluid velocity and the drag on both walls of the cylinders.
\end{abstract}

Keywords: Oscillating pressure gradient, Dean flow, Unsteady, Riemann-Sum Approximation (RSA)

\section{Background}

Research work on unsteady fully developed laminar flow attributed to circumferential pressure gradient (unsteady Dean flow) and oscillating pressure gradient has remained very active in the past decade due its increasing applications in hemodynamics, biofluid mechanics, and engineering. The underlying phenomenon of fluid flow due to heat transfer has its setback as heat alters the rheological properties of fluids in most of the systems in the aforementioned applications. Consequently, it will be desirable to design systems in which the flows are driven by pressure and not convective current.

\footnotetext{
*Correspondence: daudagambo85@gmail.com

Department of Mathematics, Ahmadu Bello University, Zaria, Nigeria
}

A theoretical analysis on laminar steady flow due to constant azimuthal pressure gradient in a channel can be dated back to the work of Dean [1, 2]. Richardson and Tyler [3] and Sexl [4] undertook an investigation on the motion of a viscous and incompressible fluid induced by an oscillating pressure gradient in a straight circular pipe. In context of blood flow in the human arterial system, the fully developed flow of a viscous and incompressible fluid with imposed periodic pressure gradient in a circular pipe was examined by Womersley [5]. Uchida [6], on the other hand, gave the exact solution for the steady motion of a viscous and incompressible fluid in a circular pipe driven by pressure gradient oscillating about a non-zero frequency. Afterwards, extensive survey has been carried out both experimentally and theoretically on this field of research by many workers. In an

\section{Springer Open}

(๑) The Author(s). 2020 Open Access This article is licensed under a Creative Commons Attribution 4.0 International License, which permits use, sharing, adaptation, distribution and reproduction in any medium or format, as long as you give appropriate credit to the original author(s) and the source, provide a link to the Creative Commons licence, and indicate if changes were made. The images or other third party material in this article are included in the article's Creative Commons licence, unless indicated otherwise in a credit line to the material. If material is not included in the article's Creative Commons licence and your intended use is not permitted by statutory regulation or exceeds the permitted use, you will need to obtain permission directly from the copyright holder. To view a copy of this licence, visit http://creativecommons.org/licenses/by/4.0/. 
attempt to have an overview of this study, we shall consider the work of Seth and Jana [7], Mullin and Greated [8], Drake [9], Smith [10], and Badr [11].

Chamkha [12], using the cosine Fourier series and method of separation of variables, performed an analytical evaluation on transient flow of a viscous and electrically conducting fluid in a channel saturated with non-conducting dusty fluid particles in the presence of applied transverse magnetic field and an oscillating pressure gradient in the direction of flow. A fully developed flow due to an oscillatory pressure gradient with timedependent curvature in a tube was reported by Waters and Pedley [13]. In recent past, Ansari et al. [14] solved numerically Navier-Stokes equations responsible for the motion of a viscous and incompressible fluid through a pipe driven by an oscillatory pressure gradient.

With regard to the continuing investigation, Tsangaris et al. [15] and Tsangaris and Vlachakis [16] reported the effect of an oscillating pressure gradient on unsteady laminar fully developed flow in the region between two concentric cylinders. The exact solution for unsteady rotating flow of a generalized Maxwell fluid in an infinite straight circular cylinder with oscillating pressure gradient was proposed by Zheng et al. [17]. Unsteady fully developed flow of a rarefied gas due a harmonically oscillating pressure gradient in a straight circular tube was studied by Tsimpoukis and Valougeorgis [18].

As the trend continues to unfold and facts are emerging, the phenomenon of an oscillating timedependent pressure driven flow has not been fully understood even though experiments have been carried out and interpretation of the observable behaviors has been formulated. Jha and Yusuf [19] in an attempt to understand transient flow formation due to a steady circumferential pressure gradient (azimuthal pressure gradient) in a composite annulus, solved semi-analytically the governing momentum equations accountable for the flow in terms of modified Bessel functions. In their work, they utilized a numerical inversing technique known as RiemannSum Approximation approach (RSA) in transforming the Laplace domain solution to time domain and concluded that velocity of the fluid is an increasing function of time. Other related literatures that adopted this method of solution include the work of Jha and Odengle [20], Yusuf and Gambo [21], and Jha and Yahaya [22].

Recently, Jha and Yahaya [22, 23] adopting the same method of solution as Jha and Yusuf [19], scrutinized unsteady Dean flow of a viscous and incompressible fluid with constant pressure gradient. In their work, they considered the flow in a horizontal concentric cylinder and later extended the work to the case when the walls of the cylinder are porous in order to superimpose the radial flow. They obtained that an increasing time is desirable for an optimum velocity and skin friction (see Jha and Yahaya [22]). In addition, Jha and Yahaya [23] reported that the fluid velocity and skin friction are increasing functions of injection and time.

However, in spite of all these contributions, no research work has been done to semi-analytically examine the influence of an oscillating time-dependent pressure gradient on Dean flow. Although, there is a general agreement that the annular effects are influenced by time, frequency, and amplitude of the oscillating pressure gradient. However, other facets like the behavior of fluid in the annular gap with an increasing time at fixed frequency and amplitude were not elucidated. The aim of this article is to extend the work of Jha and Yahaya [22] by considering an oscillating timedependent pressure gradient in addition to the azimuthal pressure gradient. The governing momentum equations are solved analytically in Laplace domain, and the Laplace domain solution is transformed to time domain using a numerical inversing technique known as Riemann-Sum Approximation (RSA). Comparison with previously established results is made at special case.

\section{Methods}

Unsteady fully developed laminar circumferential flow of a viscous and incompressible fluid in an infinite horizontal concentric cylinder is considered. It is assumed the cylinders are fixed and the fluid is Newtonian. The $Z^{\prime}$-axis is taken as axis of the cylinder in the horizontal direction. The radii of the inner and outer cylinder are $r_{1}$ and $r_{2}$, respectively (see Fig. 1). Initially, at time $t^{\prime} \leq 0$, it is assumed that the fluid is at rest. At $t^{\prime}>0$, the flow is set in motion by the applied circumferential pressure gradient $\left(\frac{\partial P}{\partial \phi}\right)$ and the oscillating time-dependent pressure gradient in the direction of flow. The Navier-Stokes and continuity equations for unsteady fully developed $\left(u_{r^{\prime}}^{\prime}=0\right)$ flow of viscous incompressible fluid written in polar coordinate systems as functions of time and radial coordinates. The following partial differential equations are given as follows:

$$
\begin{aligned}
& \frac{\partial\left(r^{\prime} u_{r^{\prime}}^{\prime}\right)}{\partial r^{\prime}}+\frac{\partial u^{\prime}}{\partial \phi}=0 \\
& \rho\left(\frac{\partial u_{r^{\prime}}^{\prime}}{\partial t^{\prime}}+u_{r^{\prime}}^{\prime} \frac{\partial u_{r^{\prime}}^{\prime}}{\partial r^{\prime}}+\frac{u^{\prime}}{r^{\prime}} \frac{\partial u_{r^{\prime}}^{\prime}}{\partial \phi}-\frac{u^{\prime 2}}{r^{\prime}}\right) \\
& =-\frac{\partial P}{\partial r^{\prime}} \\
& \quad+\mu\left(\frac{\partial^{2} u_{r^{\prime}}^{\prime}}{\partial r^{\prime 2}}+\frac{1}{r^{\prime}} \frac{\partial u_{r^{\prime}}^{\prime}}{\partial r^{\prime}}+\frac{1}{r^{\prime}} \frac{\partial^{2} u^{\prime}}{\partial \phi^{2}}-\frac{u_{r^{\prime}}^{\prime}}{r^{\prime 2}}-\frac{2}{r^{\prime 2}} \frac{\partial u^{\prime}}{\partial \phi}\right)
\end{aligned}
$$




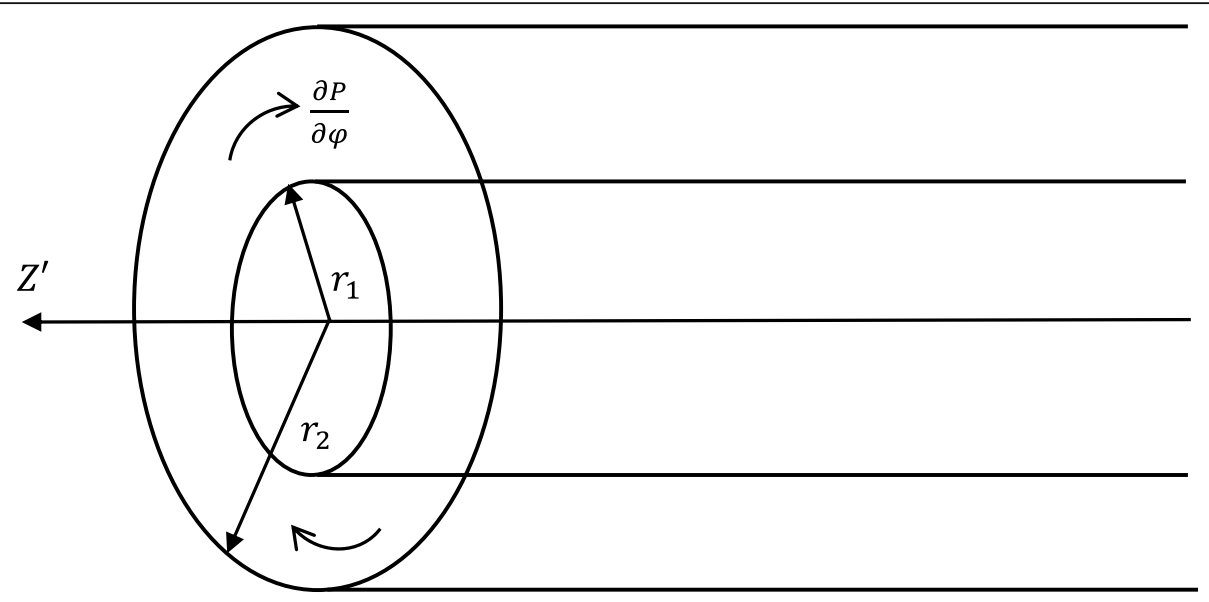

Fig. 1 Flow configuration and coordinate system

$$
\begin{aligned}
& \rho\left(\frac{\partial u^{\prime}}{\partial t^{\prime}}+u_{r^{\prime}}^{\prime} \frac{\partial u^{\prime}}{\partial r^{\prime}}+\frac{u^{\prime}}{r^{\prime}} \frac{\partial u^{\prime}}{\partial \phi}-\frac{u_{r^{\prime}}^{\prime} u^{\prime}}{r^{\prime}}\right) \\
& =-\frac{\cos \left(\omega_{0}^{\prime} t^{\prime}\right)}{r^{\prime}} \frac{\partial P}{\partial \phi}+ \\
& \mu\left(\frac{\partial^{2} u^{\prime}}{\partial r^{\prime 2}}+\frac{1}{r^{\prime}} \frac{\partial u^{\prime}}{\partial r^{\prime}}+\frac{1}{r^{\prime 2}} \frac{\partial^{2} u^{\prime}}{\partial \phi^{2}}-\frac{u^{\prime}}{r^{\prime 2}}+\frac{2}{r^{\prime 2}} \frac{\partial u^{\prime}}{\partial \phi}\right)
\end{aligned}
$$

As mentioned earlier, the cylinder is of infinite length and the flow is fully developed, thus

$$
u_{r^{\prime}}^{\prime}=0
$$

Following the approach of Tsangaris and Vlachakis [16], the governing momentum equation can be written as

$$
\begin{aligned}
\rho \frac{u^{\prime 2}}{r^{\prime}}= & \frac{\partial P}{\partial r^{\prime}} \\
\rho \frac{\partial u^{\prime}}{\partial t^{\prime}}= & -\frac{\cos \left(\omega_{0}^{\prime} t^{\prime}\right)}{r^{\prime}} \frac{\partial P}{\partial \phi} \\
& +\mu\left(\frac{\partial^{2} u^{\prime}}{\partial r^{\prime 2}}+\frac{1}{r^{\prime}} \frac{\partial u^{\prime}}{\partial r^{\prime}}-\frac{u^{\prime}}{r^{\prime 2}}\right)
\end{aligned}
$$

The initial and boundary conditions under consideration for the problem are

$$
\begin{gathered}
t^{\prime} \leq 0: u^{\prime}=0 \text { for } r_{1} \leq r^{\prime} \leq r_{2} \\
t^{\prime}>0\left\{\begin{array}{l}
u^{\prime}=0 \text { at } r^{\prime}=r_{1} \\
u^{\prime}=0 \text { at } r^{\prime}=r_{2}
\end{array}\right.
\end{gathered}
$$

Equations (5)-(7) has been rendered dimensionless using the following dimensionless quantities
$\frac{R=\frac{r^{\prime}}{r_{1}} ; \lambda=\frac{r_{2}}{r_{1}} ; \quad t=\frac{v t^{\prime}}{r_{1}^{2}} ; \quad U=\frac{u^{\prime}}{U_{0}} ; U_{0}=-r_{1} \frac{\partial P}{\partial \phi} A_{0}}{\rho v} ; \omega=\frac{r_{1}^{2} \omega^{\prime} 0}{v}$

Equations (5) and (6) can be written in the dimensionless form as

$$
\frac{\partial U}{\partial t}=\frac{\cos (\omega t)}{R}+\left(\frac{\partial^{2} U}{\partial R^{2}}+\frac{1}{R} \frac{\partial U}{\partial R}-\frac{U}{R^{2}}\right)
$$

Subject to the following initial and bound boundary conditions

$$
\begin{gathered}
t \leq 0: U=0 \text { for } 1 \leq R \leq \lambda \\
t>0 \begin{cases}U=0 & \text { at } R=1 \\
U=0 & \text { at } R=\lambda\end{cases}
\end{gathered}
$$

Employing the classical Laplace transform technique, Eqs. (9) and (10) are transformed to the Laplace domain using $\bar{U}(R, s)$ $=\int_{0}^{\infty} U(R, t) e^{-s t} d t$ where $s$ is the Laplace parameter and $(s>$ $0)$. Equations (9) and (10) in the Laplace domain are given as

$$
\frac{d^{2} \bar{U}}{d R^{2}}+\frac{1}{R} \frac{d \bar{U}}{d R}-\left(1+s R^{2}\right) \frac{\bar{U}}{R^{2}}=-\frac{s}{R\left(s^{2}+\omega^{2}\right)}
$$

Under the no slip boundary condition

$$
\left\{\begin{array}{l}
\bar{U}=0 \text { at } R=1 \\
\bar{U}=0 \text { at } R=\lambda
\end{array}\right.
$$

Following the work of Tsangaris et al. [15], the linear non-homogeneous differential equation in Eq. (11) can be reduced using the given transformation below

$$
\bar{U}(R, s)=\bar{U}_{h}(R, s)+\frac{1}{R\left(s^{2}+\omega^{2}\right)}
$$

where $\bar{U}_{h}(R, s)$ is the homogeneous solution of Eq. (11). 
Thus, employing Eq. (13), the exact solution of Eq. (11) in the Laplace domain subject to boundary conditions (12) is given below

$$
\begin{aligned}
\bar{U}(R, s)= & B_{1} I_{1}(R \sqrt{s})+B_{2} K_{1}(R \sqrt{s}) \\
& +\frac{1}{R\left(s^{2}+\omega^{2}\right)}
\end{aligned}
$$

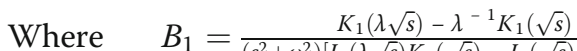
$=\frac{\lambda^{-1} I_{1}(\sqrt{s})-I_{1}(\lambda \sqrt{s})}{\left(s^{2}+\omega^{2}\right)\left[I_{1}(\lambda \sqrt{s}) K_{1}(\sqrt{s})-I_{1}(\sqrt{s}) K_{1}(\lambda \sqrt{s})\right]}$

The skin frictions at the outer surface of the inner cylinder and the inner surface of the outer cylinder are derived by differentiating Eq. (14) at $R=1$ and $R=\lambda$, respectively

$$
\begin{aligned}
\overline{\tau_{1}}(R, s) & =\left.R \frac{d}{d R}\left(\frac{\bar{U}(R, s)}{R}\right)\right|_{R=1} \\
& =\sqrt{s}\left[B_{1} I_{2}(\sqrt{s})-B_{2} K_{2}(\sqrt{s})\right]-\frac{2}{\left(s^{2}+\omega^{2}\right)} \\
\overline{\tau_{\lambda}}(R, s) & =\left.R \frac{d}{d R}\left(\frac{\bar{U}(R, s)}{R}\right)\right|_{R=\lambda} \\
& =\sqrt{s}\left[B_{1} I_{2}(\lambda \sqrt{s})-B_{2} K_{2}(\lambda \sqrt{s})\right]-\frac{2}{\lambda^{2}\left(s^{2}+\omega^{2}\right)}
\end{aligned}
$$

The vorticity of the fluid in the annular gap is given by

$$
\begin{aligned}
\bar{\eta}(R, s) & =\frac{1}{R} \frac{d}{d R}(R \bar{U}(R, s)) \\
& =\sqrt{s}\left[B_{1} I_{2}(R \sqrt{s})-B_{2} K_{2}(R \sqrt{s})\right]-\frac{2}{R^{2}\left(s^{2}+\omega^{2}\right)}
\end{aligned}
$$

It is relevant to note that the above Laplace domain solutions in Eq. (14)-(17) are to be transformed in order to determine the velocity, skin frictions and vorticity in time domain. Due to the intricate nature of the closed-form solutions, a numerical inversing procedure known as Riemann-Sum Approximation (RSA) employed by Jha and Odengle [20], Yusuf and Gambo [21], and Jha and Yahaya [22, 23] which is remarkable for its precision has been utilized in transforming Eq. (14)-(17) to time domain as follows:

$$
Z(R, t)=\frac{e^{\varepsilon t}}{t}\left[\frac{\bar{Z}(R, s)}{2}+\operatorname{Re}\left(\sum_{n=1}^{Q} \bar{Z}\left(R, \varepsilon+\frac{i n \pi}{t}\right)(-1)^{n}\right]\right.
$$

Where $Z$ constitutes $U, \tau$ or $\eta$ as the case may be, $R e$ is the real part of the summation, $i=\sqrt{-1}$ the imaginary number, $Q$ is the number of terms involve in the summation, and $\varepsilon$ is the real part of the
Bromwich contour that is used in inverting Laplace transform. The Riemann-Sum Approximation (RSA) for the Laplace inversion involves a single summation for the numerical computation, of which its exactness is dependent on the value of $\varepsilon$ and the truncation error prescribed by $Q$. Following Tzou [24], taking $\varepsilon t$ to be 4.7 gives the most desirable result.

In order to ascertain the validity of the present analysis, comparison with previously established results has been tabulated when the frequency of the oscillating time-dependent pressure gradient is taken as $0(\omega=0)$ Tables 1 and 2

\section{Results}

In an attempt to understand the influence of time, frequency of oscillating time-dependent pressure gradient and the annular gap on the velocity, skin frictions and vorticity, a MATLAB program has been written to compute and generate line graphs and numerical values for the velocity, skin friction and vorticity. The present parametric study has been performed over a reasonable range of values $0.02 \leq t \leq 0.2$ with $t=0.05$ taken as reference point, $0 \leq \omega \leq 10 \pi, \omega=\pi$ taken as reference point and $\lambda=$ 2.0. The effects of the various dimensionless parameters on the flow formation are depicted graphically

\begin{tabular}{|c|c|c|c|}
\hline \multirow[b]{2}{*}{ t } & \multirow[b]{2}{*}{$\lambda$} & \multicolumn{2}{|l|}{ Velocity profile } \\
\hline & & Present work RSA $(\omega=0)$ & $\begin{array}{l}\text { Jha and Yahaya [22] } \\
\text { exact solution }\end{array}$ \\
\hline \multirow[t]{4}{*}{0.2} & 1.2 & 0.0530 & 0.0600 \\
\hline & 1.4 & 0.0707 & 0.0813 \\
\hline & 1.6 & 0.0645 & 0.0745 \\
\hline & 1.8 & 0.0402 & 0.0460 \\
\hline \multirow[t]{4}{*}{0.4} & 1.2 & 0.0591 & 0.0600 \\
\hline & 1.4 & 0.0800 & 0.0813 \\
\hline & 1.6 & 0.0733 & 0.0745 \\
\hline & 1.8 & 0.0453 & 0.0460 \\
\hline \multirow[t]{4}{*}{ SS } & 1.2 & 0.0600 & 0.0600 \\
\hline & 1.4 & 0.0813 & 0.0813 \\
\hline & 1.6 & 0.0745 & 0.0745 \\
\hline & 1.8 & 0.0460 & 0.0460 \\
\hline
\end{tabular}
in Figs. 2, 3, 4, 5, 6, 7, 8, 9, 10, 11, 12, and 13. The combined action of a growing time, an increasing frequency of oscillating time-dependent pressure gradient and constant pressure gradient on the azimuthal velocity profile are shown in Figs. 2, 3, and 4. The effects of the

Table 1. Comparison of the present results obtained using the Riemann-Sum Approximation approach (RSA) with those of Jha and Yahaya [22] for the transient state velocity 
Table 2 Comparison of the present results obtained using the Riemann-Sum Approximation approach (RSA) with those of Jha and Yahaya [22] for the transient state skin frictions

\begin{tabular}{|c|c|c|c|c|c|}
\hline \multirow[b]{2}{*}{ t } & \multirow[b]{2}{*}{$\lambda$} & \multicolumn{2}{|l|}{ Skin friction at $R=1$} & \multicolumn{2}{|l|}{ Skin friction at $R=\lambda$} \\
\hline & & Present work RSA $(\omega=0)$ & $\begin{array}{l}\text { Jha and Yahaya [22] } \\
\text { exact solution }\end{array}$ & Present work RSA $(\omega=0)$ & $\begin{array}{l}\text { Jha and Yahaya [22] } \\
\text { exact solution }\end{array}$ \\
\hline \multirow[t]{4}{*}{0.2} & 1.2 & 0.0968 & 0.0967 & 0.0857 & 0.0856 \\
\hline & 1.4 & 0.1871 & 0.1870 & 0.1496 & 0.1495 \\
\hline & 1.6 & 0.2706 & 0.2713 & 0.1982 & 0.1987 \\
\hline & 1.8 & 0.3390 & 0.3502 & 0.2292 & 0.2376 \\
\hline \multirow[t]{4}{*}{0.4} & 1.2 & 0.0968 & 0.0967 & 0.0858 & 0.0856 \\
\hline & 1.4 & 0.1871 & 0.1870 & 0.1496 & 0.1495 \\
\hline & 1.6 & 0.2714 & 0.2713 & 0.1988 & 0.1987 \\
\hline & 1.8 & 0.3499 & 0.3502 & 0.2373 & 0.2376 \\
\hline \multirow[t]{4}{*}{ SS } & 1.2 & 0.0967 & 0.0967 & 0.0856 & 0.0856 \\
\hline & 1.4 & 0.1870 & 0.1870 & 0.1495 & 0.1495 \\
\hline & 1.6 & 0.2713 & 0.2713 & 0.1987 & 0.1987 \\
\hline & 1.8 & 0.3502 & 0.3502 & 0.2376 & 0.2376 \\
\hline
\end{tabular}

controlling parameters on skin friction on the outer surface of the inner cylinder and inner surface of the outer cylinders are depicted in Figs. 5, 6, and 7 and Figs. 8, 9, 10, respectively. The rotation of fluid produced by the azimuthal pressure in the annular gap when the velocity is acted upon by time, frequency of oscillation, and constant pressure gradient are represented by Figs. 11, 12, and 13.

\section{Discussion}

Figure 2 shows the velocity profile as time passes for a fixed frequency of oscillation. It is observed that as time increases, the velocity gradually increases as it attains steady state. On the other hand, the velocity profile for different values of the frequency of the oscillating time-dependent pressure gradient and a fixed value of time is presented in Fig. 3. It is evident from Fig. 3 that as the frequency of oscillation increases harmonically, the velocity of the fluid decreases. This is ascribed to the fact that the oscillating pressure gradient is an increasing function of frequency alone, and its increase further reduces the fluid velocity in the annular gap.

Figure 4 exhibits the influence of time on a constant circumferential pressure gradient when the frequency of the oscillation is taken as 0 . It is noted that as time passes, the fluid velocity is enhanced.

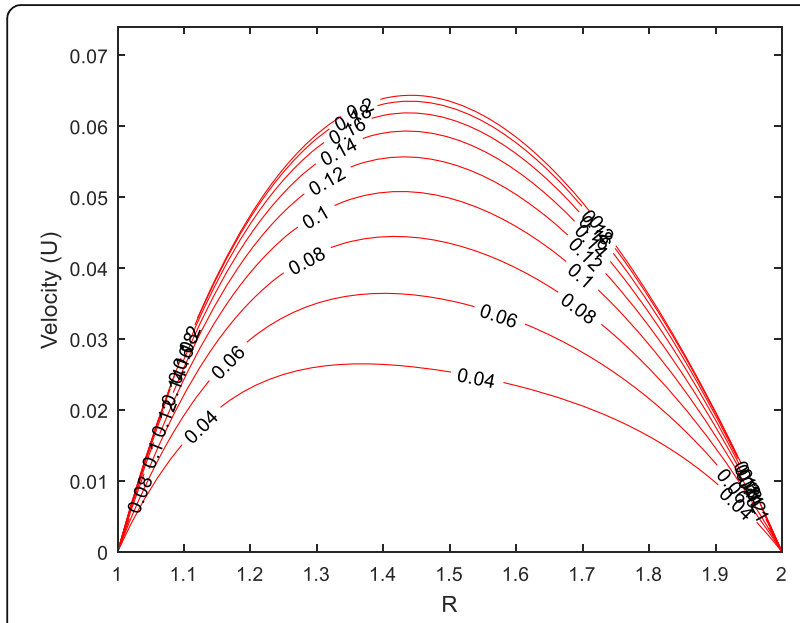

Fig. 2 Velocity profile versus time $t$ at $\omega=\pi$

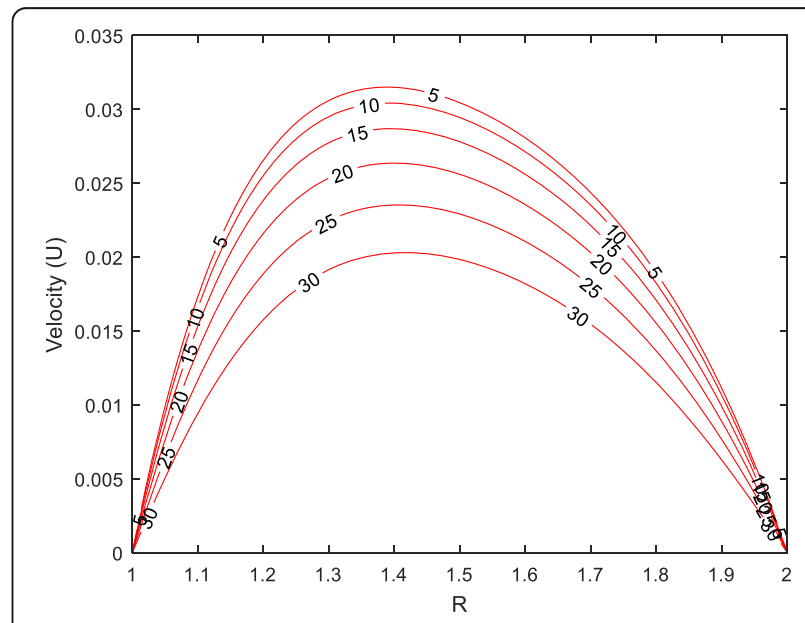

Fig. 3 Velocity profile versus frequency $\omega$ at $t=0.05$ 


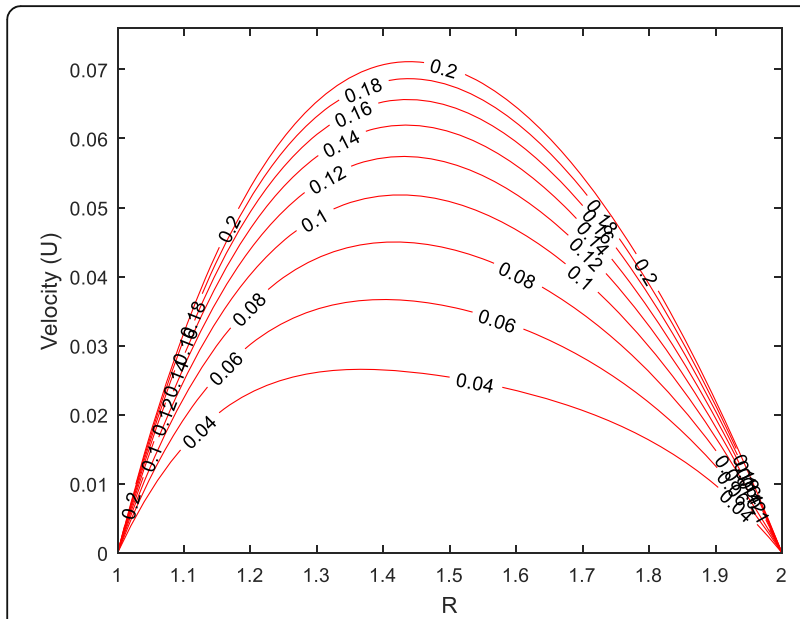

Fig. 4 Velocity profile versus time $t$ as $\omega=0$

The distribution of skin friction at the outer wall of the inner cylinder for different values of time and frequency of the oscillating time-dependent pressure gradient are shown in Figs. 5 and 6. It is clear from Fig. 5 that as time passes with a fixed frequency of oscillation, the skin friction increases and later drops with further distance from the wall. It is interesting to note that the reverse trend is observed as time is fixed and the frequency of the oscillating time-dependent pressure gradient increases as depicted in Fig. 6.This is due to the fact that as the frequency increases harmonically, the fluid velocity gradually drops and consequently weakening the skin friction on the wall.

Figure 7 shows the influence of time on the skin friction at the outer surface of the inner cylinder with an applied constant pressure gradient. It is seen from Fig. 7 that the

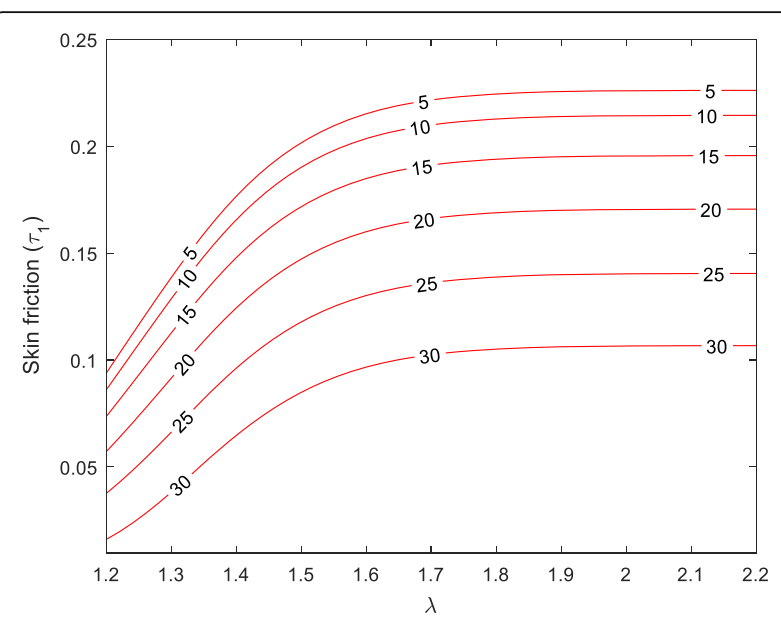

Fig. 6 Distribution of skin friction versus frequency $\omega$ at $R=1, t=0.05$

effect of time and a constant pressure gradient is to increase skin friction at the outer wall of the inner cylinder.

Figure 8 shows the effect of time with a fixed frequency of oscillation on skin friction at the inner wall of the outer cylinder. It is observed that as time passes, the skin friction increases attaining its maximum and drops gradually towards the wall.

The variation of skin friction at the inner wall of the outer cylinder with a fixed time and an increasing frequency of the oscillating time-dependent pressure gradient is demonstrated in Fig. 9. It is seen that the skin friction decreases as the frequency of the oscillating timedependent pressure gradient is increased. In addition, the decreased is more pronounced on the walls of the cylinder. On the other hand, influence of time on the skin friction as the frequency of the oscillating time-dependent

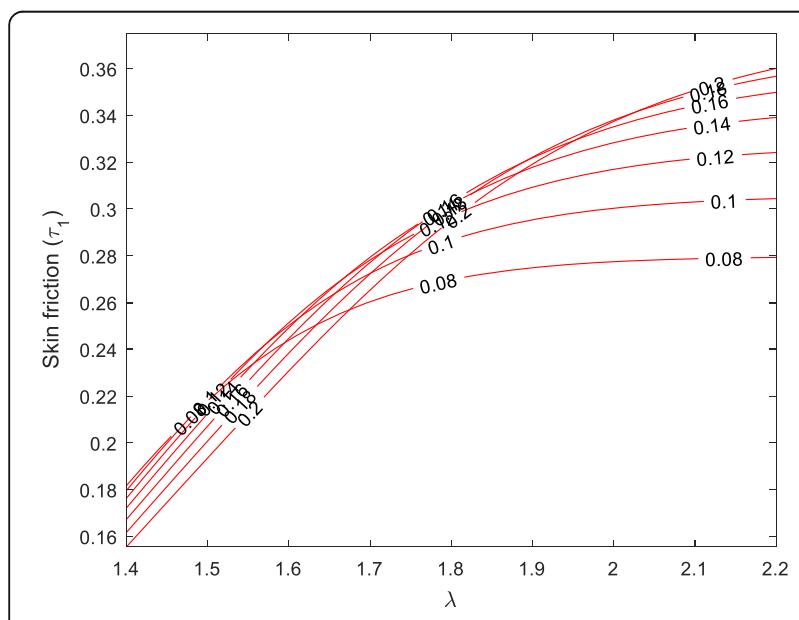

Fig. 5 Distribution of skin friction versus time at $R=1, \omega=\pi$

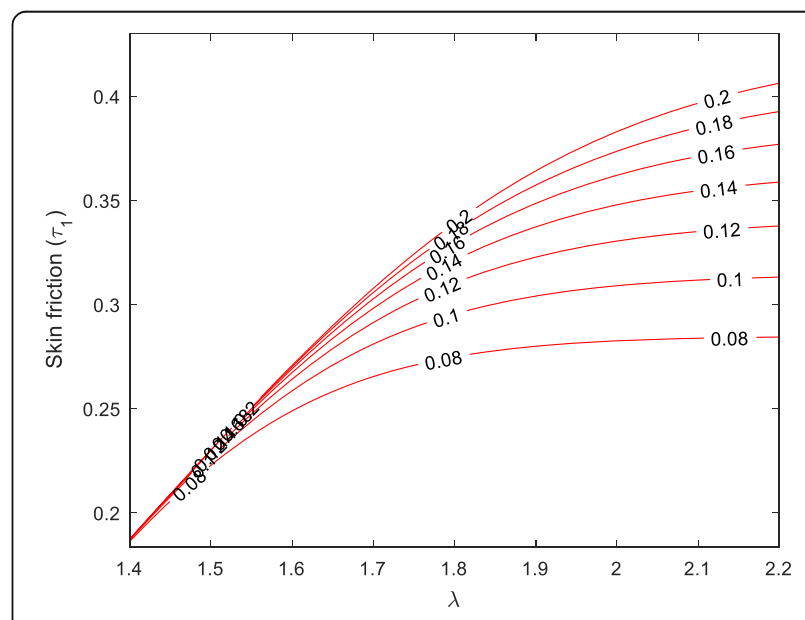

Fig. 7 Distribution of skin friction versus time $t$ at $R=1, \omega=0$ 


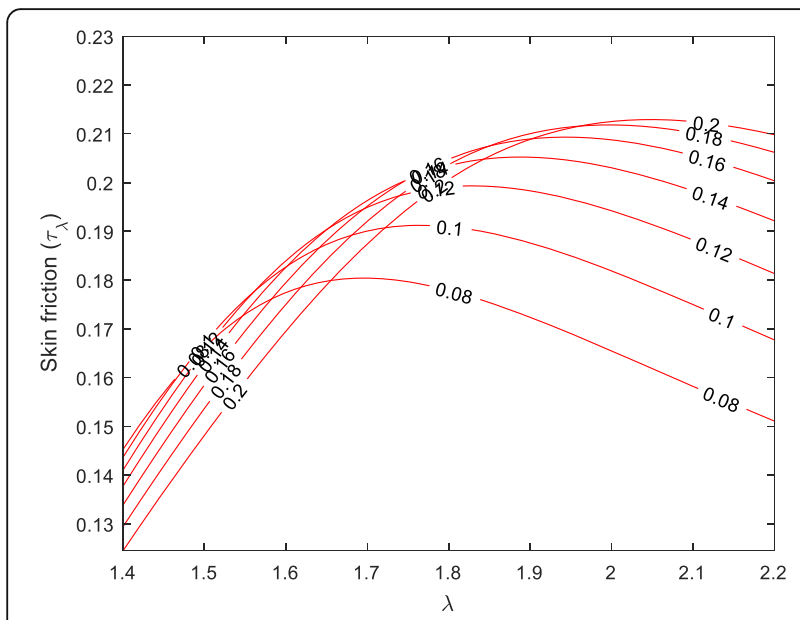

Fig. 8 Distribution of skin friction versus time $t$ at $R=\lambda, \omega=\pi$

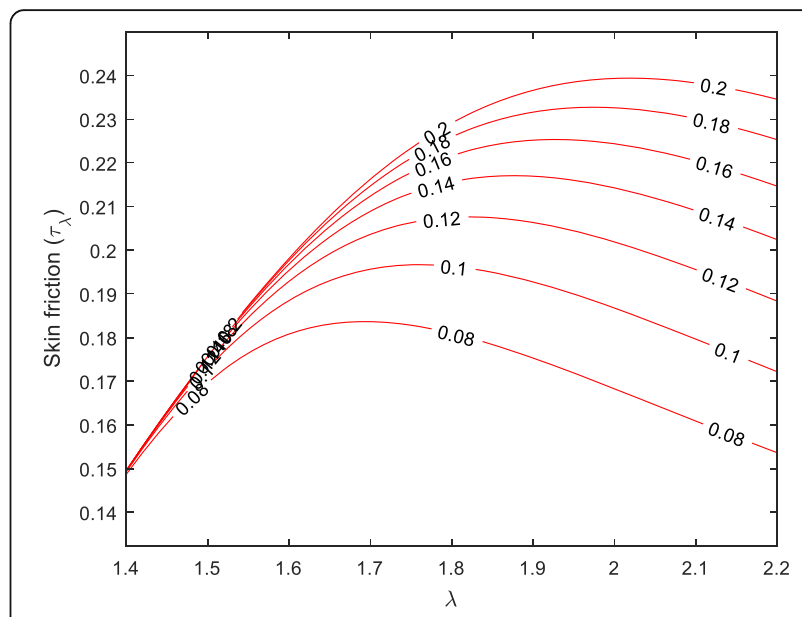

Fig. 10 Distribution of skin friction versus time $t$ at $R=\lambda, \omega=0$ pressure gradient is taken as 0 is presented in Fig 10. Result shows that the skin friction decreases as time passes.

The effects of frequency of oscillation and time on the fluid vorticity are shown in Figs. 11, 12, and 13. It is observed that the vorticity increases initially and subsequently weakens as time passes. Although there is a general decrease in magnitude of the vorticity with further distance towards the wall of the outer cylinder as time increases, the decrease is subtle when the frequency is no longer oscillating but rather a constant pressure gradient as evident from Fig. 13.

\section{Conclusions}

The influence of time and an oscillating timedependent pressure gradient on Dean flow has been analyzed. The closed-form solution of the governing

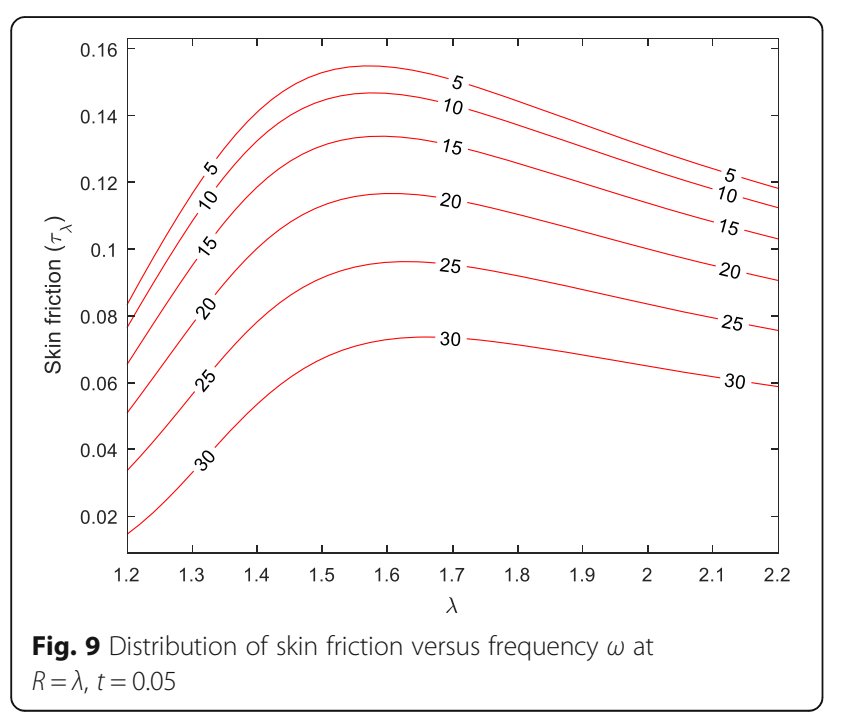

momentum equation has been derived semianalytically using the Laplace transformation technique in conjunction with Riemann-Sum Approximation (RSA) as a tool for inversion. The effect of various flow parameters on the flow formation has been represented pictorially. Findings suggest that the fluid velocity, skin frictions, and fluid vorticity can be minimized by increasing the frequency of oscillation. Furthermore, the azimuthal velocity is seen to increase steadily as time passes with an imposed frequency of oscillation, although the increase subtle as seen when a constant pressure gradient is applied.

\subsection{Nomenclature}

$A_{0}$ Amplitude of oscillating pressure gradient $r_{1}$ Radius of the inner cylinder $(m)$

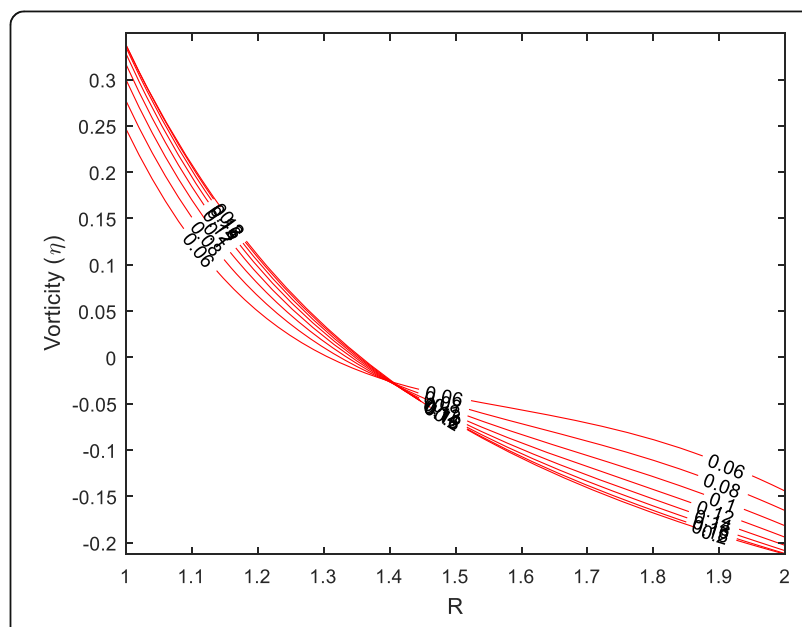

Fig. 11 Vorticity versus time $t$ at $\omega=\pi$ 


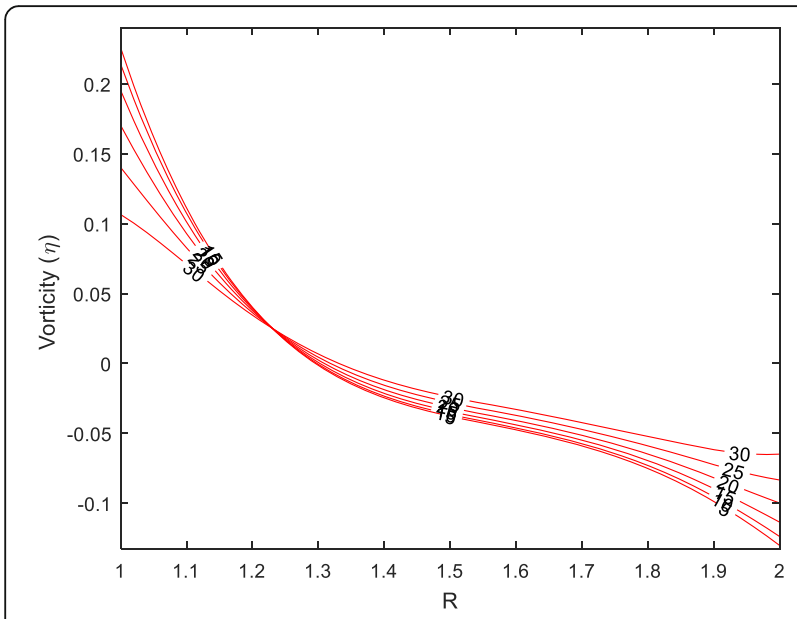

Fig. 12 Vorticity versus frequency $\omega$ at $t=0.05$

$r_{2}$ Radius of the outer cylinder $(m)$

$P$ Static pressure $\left(\mathrm{Kg} / \mathrm{ms}^{2}\right)$

$R$ Dimensionless radius

$s$ Laplace parameter

$t$ Dimensionless time $(s)$

$U_{0}$ Reference velocity $(\mathrm{m} / \mathrm{s})$

$u^{\prime}{ }^{\prime}$ Radial velocity $(\mathrm{m} / \mathrm{s})$

$u^{\prime}$ Circumferential velocity $(\mathrm{m} / \mathrm{s})$

$U$ Dimensionless velocity

\subsection{Greek letters}

$\lambda$ Radii ratio $\left(r_{2} / r_{1}\right)$

$\rho$ Fluid density $\left(\mathrm{Kg} / \mathrm{m}^{3}\right)$

$\eta$ Vorticity

$\tau$ Skin friction

$\mu$ Dynamic viscosity of the fluid $(\mathrm{Kg} / \mathrm{ms})$

$\omega$ Frequency of oscillating time-dependent pressure gradient

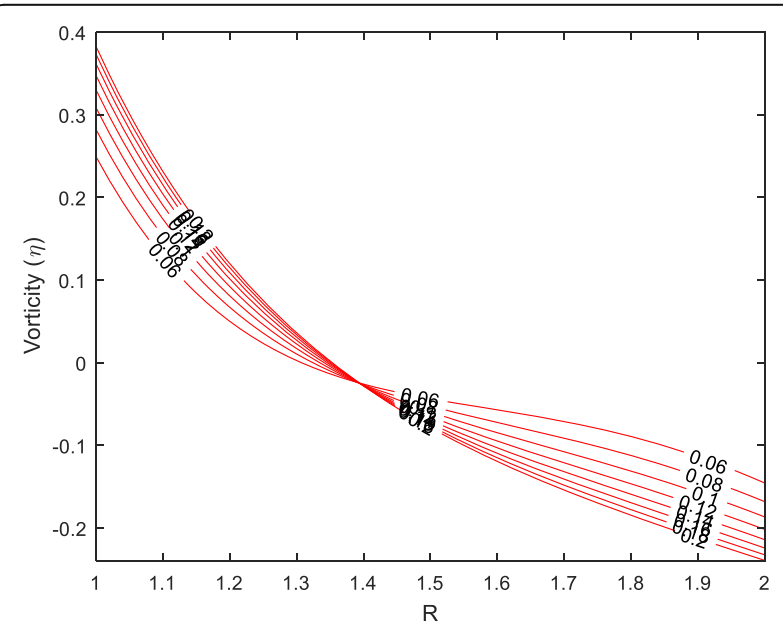

Fig. 13 Vorticity versus time $t$ at $\omega=0$

\section{Abbreviations}

RSA: Riemann-Sum Approximation; SS: Steady state

\section{Acknowledgements}

Not applicable.

\section{Authors' contributions}

BKJ performed the conceptualization, investigation, supervision, and validation of the approaches in this research work. DG was responsible for the methodology, data curation, writing-original draft preparation, writingreviewing, and editing. All authors have read and approved the manuscript.

\section{Funding}

No funding was received in the course of this research work.

\section{Availability of data and materials}

Not applicable.

Ethics approval and consent to participate

Not applicable.

\section{Consent for publication}

Not applicable.

\section{Competing interests}

The author declares that they have no competing interests.

Received: 17 April 2020 Accepted: 30 June 2020

Published online: 06 October 2020

\section{References}

1. Dean WR (1927) XVI. Note on the motion of fluid in a curved pipe. The London, Edinburgh, and Dublin Philosophical Magazine and Journal of Science 4(20):208-223. https://doi.org/10.1080/14786440708564324

2. Dean WR (1928) Fluid motion in a curved channel. In Proceeding Royal Soc Lond A: Math Phys Eng Sci 121:402-420

3. Richardson EG, Tyler E (1929) The transverse velocity gradient near the mouths of pipes in which an alternating flow is established. Proc Phys Soc Lond 42:1-15

4. Sexl T (1930) On the annular effect discovered by E.G Richardson. Z Phys 61:349-362

5. Womersley JR (1995) Method for the calculation of velocity, rate of flow and viscous drag in arteries when the pressure gradient is known. J Physiol 127:553-563

6. Uchida S (1956) The pulsating viscous flow superposed on the steady laminar motion of incompressible fluid in a circular pipe. J Appl Math 7:403-422

7. Seth GS, Jana RS (1980) Unsteady hydromagnetic flow in a rotating channel with oscillating pressure gradient. Acta Mech 37:29-41

8. Mullin T, Greated CA (1980) Oscillatory flow in curved pipes. Part 1. The developing-flow case. J Fluid Mech 98(2):383-395

9. Drake DG (1965) On the flow in a channel due to a periodic pressure gradient. Quart J of Mech and Applied Math 18(1):1-10

10. Smith FT (1975) Pulsatile flow in curved pipes. J Fluid Mech 71(1):15-42

11. Badr HM (1997) Symmetrically oscillating viscous flow over an elliptic cylinder. J of Fluids and Structures 11:745-766

12. Chamkha AJ (1997) Unsteady flow of an electrically conducting dusty-gas in a channel due to an oscillating pressure gradient. Appl Math Model 21(5): 287-292. https://doi.org/10.1016/s0307-904x(97)00018-8

13. Waters SL, Pedley TJ (1999) Oscillatory flow in a tube of time-dependent curvature. Part 1. Perturbation to flow in a stationary curved tube. J Fluid Mech 383:327-352

14. Ansari AR, Miller JJH, Shishkin Gl (2006) A robust numerical method for flow through a pipe driven by an oscillating pressure gradient. Int J Numer Methods Fluids 53(3):471-484. https://doi.org/10.1002/fld.1290

15. Tsangaris S, Kondaxakis D, Vlachakis NW (2006) Exact solution of the NavierStokes equations for the pulsating dean flow in a channel with porous walls. Int J Eng Sci 44:1498-1509

16. Tsangaris S, Vlachakis NW (2007) Exact solution for the pulsating finite gap dean flow. Appl Math Model 31:1899-1906

17. Zheng L, Li C, Zhang X, Gao Y (2011) Exact solutions for the unsteady rotating flows of a generalized Maxwell fluid with oscillating pressure gradient between coaxial cylinders. Comput Math Appl 62(3):1105-1115. https://doi.org/10.1016/j.camwa.2011.02.044 
18. Tsimpoukis A, Valougeorgis D (2017) Rarefied isothermal gas flow in a long circular tube due to oscillating pressure gradient. Microfluid Nanofluid 22(1). https://doi.org/10.1007/s10404-017-2024-2

19. Jha BK, Yusuf TS (2018) Transient pressure driven flow in an annulus partially filled with porous material: Azimuthal pressure gradient. Mathematical Modelling of Engineering Problems 5(3):260-267

20. Jha BK, Odengle JO (2014) Unsteady Couette Flow in a Composite Channel Partially Filled with Porous Material: A Semi-analytical Approach. Transp Porous Media 107(1):219-234. https://doi.org/10.1007/s11242-014-0434-0

21. Yusuf TS, Gambo D (2019) Impact of heat generation/absorption on transient natural convective flow in an annulus filled with porous material subject to isothermal and adiabatic boundaries. GEM - International J on Geomathematics 10(20):1-16 https://doi.org/10.1007/s13137-019-0132-8

22. Jha BK, Yahaya JD (2018) Transient Dean flow in an annulus: a semianalytical approach. Journal of Taibah University for Science 13(1):169-176

23. Jha BK, Yahaya JD (2019) Transient Dean flow in a channel with suction/injection: A semi-analytical approach. Journal of Process Mechanical Engineering 233(5):1-9

24. Tzou DY (1997) Macro to Microscale Heat Transfer: The Lagging Behavior. Taylor and Francis, London

\section{Publisher's Note}

Springer Nature remains neutral with regard to jurisdictional claims in published maps and institutional affiliations.

\section{Submit your manuscript to a SpringerOpen ${ }^{\odot}$ journal and benefit from:}

- Convenient online submission

- Rigorous peer review

- Open access: articles freely available online

High visibility within the field

- Retaining the copyright to your article

Submit your next manuscript at $\boldsymbol{\nabla}$ springeropen.com 